\title{
Reinterpretando la Dislexia. Propuesta diferenciadora para una neurodidáctica inclusiva
}

\author{
Iluminada Sánchez-Domenech *
}

Resumen. Hasta la aparición de las técnicas de neuroimagen las investigaciones sobre la Dislexia se centraron en el déficit fonológico como uno de los factores causales. Estas técnicas han permitido avanzar en el conocimiento anatómico-funcional de las distintas áreas del cerebro revelando la amplitud y complejidad de la red de lectura del cerebro.

Con el objetivo de una mayor compresión este trastorno del neurodesarrollo, se analizan los últimos avances sobre las funciones neuroanatómicas de las distintas áreas del cerebro implicadas en la lectura y las investigaciones sobre la etiología neurológica de la Dislexia. Se concluye con la necesidad de considerar un trastorno del neurodesarrollo sensorial localizado en áreas primarias auditiva y visual que conlleva un deficiente procesamiento de la información viso-espacio-temporal. Se considera la Dislexia, y por tanto también el déficit fonológico, como una consecuencia de este trastorno. Esta diferenciación permite visibilizar otros síntomas menos atendidos por la investigación y la didáctica.

Se extraen implicaciones cognitivas del déficit del procesamiento espaciotemporal en las etapas de educación infantil y primaria ante tareas típicas del aula a partir de las cuales diseñar una didáctica inclusiva, comprensiva con la naturaleza neurológica del trastorno, que no penalice a los alumnos que padecen este trastorno neurológico.

Palabras clave: conciencia fonológica; deficiencia sensorial; dificultades de lectura y escritura; Dislexia; trastorno neurológico.

\section{REINTERPRETANDO A DISLEXIA. PROPOSTA DIFERENCIADA PARA UMA NEU- RODIDÁTICA INCLUSIVA}

Resumo. Até o surgimento das técnicas de neuroimagem, as pesquisas sobre Dislexia consideravam o déficit fonológico como um dos fatores causais. Essas técnicas possibilitaram o avanço do conhecimento anatômico e funcional das diferentes áreas do cérebro, revelando a amplitude e a complexidade da rede de leitura do cérebro.

Com o objetivo de compreender melhor este transtorno do neurodesenvolvimento, analisam-se os últimos avanços nas funções neuroanatômicas das diferentes áreas do cérebro que envolvem a leitura e as pesquisas sobre a etiologia neurológica da Dislexia. Conclui-se que a necessidade de considerar um transtorno do neurodesenvolvimento sensorial localizado em áreas auditivas e visuais primárias acarreta um processamento deficiente

\footnotetext{
* Universidad Internacional de la Rioja (España)
} 
da informação visual, espacial e temporal. A Dislexia é considerada e, portanto, também o déficit fonológico, uma consequência desse transtorno. Essa diferenciação permite visualizar outros sintomas menos abordados pela pesquisa e a didática.

Obtemos implicações cognitivas do déficit de processamento espaçotemporal nas etapas da educação infantil e do ensino fundamental diante de tarefas típicas de sala de aula para desenvolver uma didática inclusiva, compreensiva com a natureza neurológica do transtorno, que não penalize os alunos que sofrem este transtorno neurológico.

Palavras-chave: consciência fonológica; deficiência sensorial; dificuldades de leitura e escrita; Dislexia; transtorno neurológico.

\section{REINTERPRETING DYSLEXIA. A DIFFERENTIATING PROPOSAL FOR AN INCLUSIVE} NEURODIDACTICS

Abstract. Until the appearance of neuroimaging techniques, research on Dyslexia has focused on the phonological deficit as the main causal factor. These techniques have made it possible to advance the anatomical-functional knowledge of the different areas of the brain, revealing the amplitude and complexity of the brain's reading network.

In order to better understand this neurodevelopmental disorder, we analyze the latest advances on the neuroanatomical functions of the different areas of the brain involved in reading and research on the neurological etiology of dyslexia. We conclude with the need to consider a sensory neurodevelopmental disorder located in primary auditory and visual areas that entails a deficient processing of visuo-spatio-temporal information. Dyslexia is considered, and therefore also the phonological deficit, as a consequence of this disorder. This differentiation makes it possible to visualize other symptoms less addressed by research and didactics.

Cognitive implications in typical classroom tasks of the spatiotemporal processing deficit in the early childhood and primary education stages are extracted from which to design an inclusive didactics, comprehensive with the neurological nature of the disorder, which does not penalize students who suffer this neurological disorder.

Keywords: dyslexia; neurological impairments; phonological awareness; reading and writing difficulties; sensory deficiency.

\section{INTRODUCCIÓN}

El debate sobre qué es la Dislexia y cuál es su origen biológico dura ya más de 100 años. En su polémico libro "The Dislexia debate" Elliot y Grigorenko (2014) afirman que el término "Dislexia" ha generado demasiada confusión y ha dejado de ser útil tanto para fines científicos como prácticos. En consecuencia, proponen descartar el término para referirse a dificultades persistentes de lectura y utilizar en su lugar el término "reading disability" 
(discapcidad lectora). Otros autores como Vidyasagar y Pammer (2010) afines a la hipótesis del déficit viso-espacial apuestan por considerarla parte de un trastorno de la atención espacial y temporal más amplio. El déficit fonológico sigue siendo considerado por otro sector de autores el mejor predictor y origen de la Dislexia.

Según Pennington (2006), la Dislexia es un desorden multifactorial caracterizado por numerosos déficits que, combinados, tienen como resultado un déficit en la lectura y, por tanto, la investigación centrada en un único déficit como el fonológico, es demasiado simplista para explicar este "complejo desorden del neurodesarrollo" (p. 1). Por otra parte, incluir dentro de la etiqueta "Dislexia" cualquier dificultad en la lectoescritura complica la distinción entre los niños que tienen dificultades en la lectoescritura por motivos de diferente naturaleza y los niños con un trastorno del neurodesarrollo en áreas primarias visual y/o auditiva que condiciona gravemente su aprendizaje y su adaptación a la didáctica escolar. En el primero de los casos, la consecuencia es un posible sobre-diagnóstico; en el segundo, la infravaloración del problema, la intervención errónea y una didáctica que penaliza a estos alumnos.

Como reconocen Quercia, Feiss y Michel (2013, p. 870), "actualmente, seguimos sin ser capaces de distinguir claramente las causas de las consecuencias". La multiplicidad de definiciones de Dislexia con variaciones importantes entre ellas, como la de incluir o no síntomas relacionados con la coordinación visomotora o la percepción espacio-temporal, nos lleva a preguntarnos si estamos ante síntomas comunes de diferente etiología dada la amplia red de zonas implicadas en la lectura. Un trastorno del neurodesarrollo sensorial visual y/o auditivo explica el retraso o limitación en las capacidades adaptativas del niño en actividades en las que están implicadas la orientación espacio-temporal, el esquema corporal, la lateralización, la secuenciación y la coordinación visomotora (Etchepareborda, 2002) que no se explica a través del déficit fonológico como demostramos en este documento. Los resultados de los estudios neuroanatómicos y las consecuencias cognitivas de las diferencias halladas en los cerebros de los sujetos considerados disléxicos visibilizan esas otras manifestaciones que permiten diseñar una didáctica escolar coherente con la naturaleza neurológica del trastorno o, al menos, que no exija a estos alumnos aquello para lo que su cerebro todavía no ha alcanzado la maduración y la compensación necesarias abocándolos al fracaso prematuro. 


\section{EL ESTUDIO NEUROANATÓMICO DE LA DISLEXIA}

Las nuevas técnicas de neuroimagen han permitido conocer funciones neuroanatómicas de las diferentes áreas del cerebro con mayor profundidad, lo que ha llevado a ampliar la red de lectura del cerebro a otras áreas además de las relacionadas tradicionalmente. En esta red, las áreas primarias auditivas y visuales han revelado su papel primordial. Concretamente el tronco encefálico, donde se sitúa el Tálamo, centro de procesamiento de los estímulos sensoriales (gusto, equilibrio, visión y audición), ha centrado la mayoría de las investigaciones sobre la etiología neurológica de la Dislexia divididas en dos líneas de investigación que compiten por explicarla: i) la que se centra en los procesos visuales y ii) la que defiende los procesos auditivos. Existen pocos estudios longitudinales que den cuenta de los precursores neurológicos de la Dislexia, es decir, presentes antes del aprendizaje de la lectoescritura. Uno de estos estudios es el de Clark, Helland, Specht, Narr, Manis, Toga y Hugdahl (2014). Los niños que desarrollaron más tarde Dislexia tenían una corteza más delgada en varias regiones del hemisferio izquierdo: giro de Heschl, giro lingual, circunvolución frontal medial, circunvolución cingulada media y un área en la corteza orbitofrontal derecha (centro de asociación de estímulos sensoriales; Rolls, 2004). Sin embargo, las diferencias previas a la lectura se identificaron en las áreas de procesamiento sensorial de menor nivel auditivas y visuales.

\subsection{Breve aproximación a la investigación sobre la neuroanatomía del procesamiento visual y su relación con la Dislexia}

En un estudio longitudinal Ilevado a cabo por Franceschini, Gori, Ruffino, Pedrolli y Facoetti (2012) la atención visoespacial resultó ser buen predictor del rendimiento en el aprendizaje de la lectura. Vidyasagar y Pammer (2010) defienden la hipótesis de un déficit en la atención viso-espacial como causa de la Dislexia situando el origen la misma en algún punto localizado entre la retina y el córtex parietal, es decir, a lo largo de la vía dorsal. Según los autores, el resultado cognitivo es un déficit en el reconocimiento correcto de la secuencia de letras por un mecanismo atencional, el foco de atención, que busca los atributos relevantes de un objeto para poder reconocerlo enlazando sus atributos (forma, colores, profundidad, movimiento y tamaño) y su situación espacial relativa. Consideran el déficit fonológico una consecuencia y no la causa de la Dislexia. Un argumento a favor de esta hipótesis, como ellos mismos señalan, es que la dificultad para determinar la secuencia de las letras que conforman las palabras (omisiones, adiciones, sustituciones) y la rotación de letras no puede explicarse a través del déficit fonológico. Las palabras se reconocen secuencialmente, con solo una o algunas letras se procesan a la vez por el sistema de reconocimiento de objetos y esta se- 
cuencia temporal conserva la secuencia espacial y la dirección de las letras, ordenadas de izquierda derecha. Por tanto, el orden en el que se ven y oyen las letras sirve al cerebro para almacenar en la memoria la secuencia invariable de las letras que conforman la palabra permitiendo su reconocimiento y recuperación. Para entender esta teoría nos aproximarnos a la neuroanatomía del procesamiento visoespacial en el cerebro.

Mishkin, Ungerleider y Macko (1983) fueron los primeros en localizar en sus experimentos con macacos dos vías perceptuales paralelas: la vía dorsal y la vía ventral. La primera, la vía dorsal, representa el "dónde"; conecta a través del lóbulo occipital con el lóbulo parietal inferior. Sus funciones son: localización del movimiento, representación de relaciones espaciales y control de los movimientos oculares y extremidades. Estas proyecciones occipitoparietales conectan con el sistema límbico y la corteza frontal, que hacen posible la construcción de mapas mentales y la guía visual de los actos motores.

La vía ventral va desde la corteza visual primaria y secundaria hacia la corteza temporal inferior; representa el "qué" o identificación y procesamiento del objeto en sí, su forma, color, textura, tamaño y detalles pictóricos y consiste en un sistema de proyecciones que interconecta áreas del estriado, preestriado y áreas temporales inferiores. Las proyecciones continúan hacia el lóbulo temporal y la región ventral del lóbulo frontal, lo que permite asociar los objetos con otros eventos y con los actos motores y emocionales correspondientes. Según Twomey, Kawabata, Price, Devlina (2011), esta vía actúa como una interfaz que vincula la forma visual con el procesamiento no visual tanto en direcciones ascendentes como descendentes. Esto es, esta región hace más que retrasmitir información visual hacia el sistema del lenguaje, integra de manera interactiva las señales visuales ascendentes con información de orden superior (léxicas y probablemente semánticas) de arriba hacia abajo que no están presentes en los estímulos visuales, influyendo así en su proceso de reconocimiento.

Kravitz, Kadharbatcha, Baker y Mishkin (2011) proponen una nueva estructura neuronal, complementaria al modelo de las dos vías, basada en la identificación de una trifurcación de la vía dorsal. Afirman que es mucho más compleja y con más funciones implicadas de lo que se conocía hasta el momento y que podría considerarse más un "cómo" que un "dónde"; estaría relacionada con la transmisión automática guiada visualmente no consciente. Algunas características funcionales relevantes de esas subregiones localizadas a lo largo de los 3 circuitos descritos son las que siguen:

- El circuito occipito-parietal, transforma las representaciones visuales en referencias a las partes del cuerpo y el ojo y regula la 
iniciación y el control de los movimientos oculares (movimientos sacádicos) que son cruciales para la memoria de trabajo espacial.

- Las áreas asociadas al circuito parietal premotor mantienen coordinados los mapas del espacio y la posición del cuerpo, coordinación necesaria para la acción guiada visualmente en el espacio peripersonal. Las subregiones de este circuito reciben información de regiones del cerebelo que proporcionan información vestibular sobre la posición de los brazos, el ojo y la cabeza, contiene representaciones tridimensionales de los objetos para guiar los movimientos de alcance y agarre y reciben información del córtex auditivo. Además esta región está ampliamente involucrada en la memoria a largo plazo y la recuperación de ambas modalidades sensoriales, auditiva y visual. La actividad en esta zona parietal aumenta con demandas de búsqueda atencional selectiva.

- El circuito parietal medial es el más complejo de los circuitos e incluye al hipocampo. También es el menos estudiado en humanos. Contiene representaciones espaciales de escenas que son principalmente alocéntricas, información que es vital para la navegación.

Gori, Seitz, Ronconi, Franceschini y Facoetti (2016) señalan que la vía dorsal identifica y selecciona regiones relevantes en el espacio para luego pasarlas a la vía ventral, es decir, la dorsal guía a la ventral. Por lo tanto, un déficit de la función de la vía dorsal, puede tener un efecto cascada en todos los procesos cognitivos sucesivos. Comenzamos el recorrido por esta cascada neurocognitiva en la zona de donde parten ambas vías, el tálamo.

Las recientes investigaciones sobre el tálamo visual han revelado que su rol en las funciones cognitivas es mucho más comprehensiva de lo que se creía, resultando ser una estructura clave en la memoria, la atención, el control de impulsos, la toma de decisiones, el monitoreo interno de las acciones, la percepción espacial, la selección de planificación y respuesta y la coordinación visomotora (Mitchell, Sherman, Sommer, Mair, Vertes et al, 2014). El Pulvinar, la parte más voluminosa del tálamo, realiza una importante función topográfica, discrimina la posición del objeto codificando con precisión la información espacial (Fischer y Whitney, 2009) que es una de las operaciones fundamentales que el sistema visual tiene que realizar para integrar información visual de varias dimensiones de características.

Un área importante del tálamo visual es el núcleo geniculado lateral (NGL) de donde parten las vías magno y parvocelular (Ilustración 1). El sistema magnocelular permite un procesamiento rápido de la información, es 
ciego al color, procesa la información referente a la profundidad, la forma y movimiento del estímulo. Las células parvo procesan el color y los detalles finos. El $90 \%$ de los imputs que recibe la vía dorsal proceden del magnocelular y solo el $10 \%$ proceden del parvocelular (Stein, 2018). Entre las múltiples funciones que realiza se encuentra la construcción de un marco de referencia centrado en la cabeza, un primer paso para obtener un marco egocéntrico, es decir, relaciona lo que está afuera con nuestra propia posición. También realiza funciones oculomotoras relacionadas con los movimientos sacádicos (Weyand, 2015).

\section{ILUSTRACIÓN 1}

\section{El núcleo geniculado lateral (NGL) y las vías magno y parvocelular}

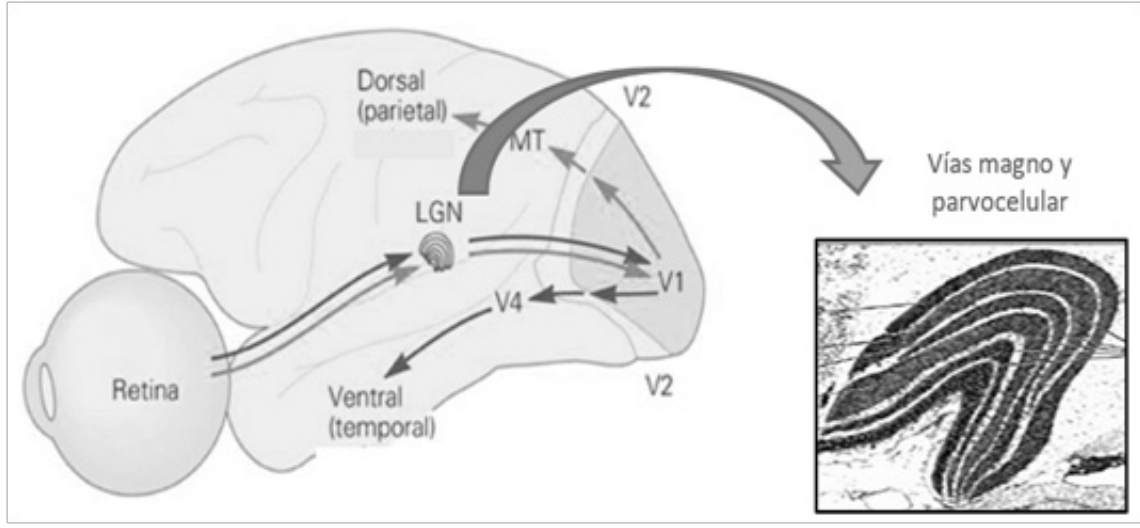

Livingstone, Rosen, Drislane, y Galaburda (1991) encontraron deficiencias fisiológicas y anatómicas en el sistema magnocelular en disléxicos. Este hallazgo generó una de las hipótesis más influyentes sobre el origen neurológico de la Dislexia.

Müller-Axt, Anwander y Von Kriegstein (2017) descubrieron que los individuos con Dislexia tienen conexiones estructurales reducidas en la vía directa entre el tálamo visual izquierdo (NGL) y el área temporal media izquierda (MT en Ilustración 1), es decir, la interrupción se produciría en un nivel más primario. Esta área conecta con el lóbulo parietal y es crítica para la percepción del movimiento visual (Gori, Seitz, Ronconi, Franceschini y Facoetti, 2016) y con las habilidades RAN (Rapid Automatized Naming), déficits clave en la Dislexia. Ben-Shachar, Dougherty, Deutsch, y Wandell, (2007) encontraron una fuerte correlación de la actividad MT con la conciencia fonológica y una correlación más débil con la habilidad RAN. Según Bueti, Bahrami y Walsh (2008), el lóbulo parietal posterior es importante para la temporalización de los estímulos auditivos y visuales y él área MT es necesaria sólo para la temporalización de los eventos visuales. 
Giraldo-Chica, Hegarty y Schneider (2015) encontraron que el NGL izquierdo era significativamente más pequeño en volumen en sujetos con Dislexia y también difería en la forma; no se observaron diferencias en la NGL derecha. Según los autores, estos resultados son consistentes con la teoría de un déficit en el sistema magnocelular y apoyan las teorías de la Dislexia que implican diferencias en el sistema visual anterior.

Dos áreas a destacar del circuito occipital (área visual primaria) y que reciben información directa del NGL a través del sistema magno y parvocelular, son el giro fusiforme, especializado en el procesamiento de palabras impresas (Finn, Shend, Holahan, Scheinost, Lacadie et al, 2014) y el giro lingual, involucrado en el procesamiento semántico (Ghosh, Basu, Kumaran y Khushu, 2010) y en la codificación de memorias visuales. Con respecto a esta última función, Kozlovskiy, Pyasik, Korotkova, Vartanov, Glozman y Kiselnikov (2014) encontraron activación en esta área relacionada con la percepción y la memoria de trabajo para estímulos visuales con cierta especialización hemisférica para distintos tipos de estímulos (por ej. caras impresas de bajo nivel visual, figuras geométricas o letras). Clark et al (2014) encontraron diferencias de grosor en esta área en el hemisferio izquierdo en niños que más tarde desarrollaron Dislexia, es decir, antes del aprendizaje de la lectura.

La cascada continúa hacia el lóbulo parietal inferior. Las dos principales subdivisiones de esta área son el giro supramarginal y el giro angular, que se han relacionado con aspectos del procesamiento fonológico y semántico, respectivamente. Concretamente el giro supramarginal está involucrado con el reconocimiento automático visual de la palabra (Stoeckel, Gough, Watkins y Devlin, 2009). Lobier, Peyrin, Pichat, Le Bas y Valdois (2014), observaron la activación del lóbulo parietal en lectores normales y disléxicos en tareas no verbales de reconocimiento de cadenas de caracteres numéricos y alfanuméricos. Mientras que los lectores normales activaron las áreas parietales con mayor fuerza para el procesamiento múltiple de elementos individuales (lado derecho para alfanumérico y bilateral para no alfanumérico), las activaciones parietales derechas más fuertes estaban ausentes para los participantes disléxicos. En lectores disléxicos observaron una actividad significativamente reducida del lóbulo supraparietal derecho y en el lóbulo occipito-temporal ventral independientemente del tipo de estímulo.

\subsection{Breve aproximación a la investigación sobre la neuroanatomía del procesamiento auditivo y su relación con la Dislexia}

El aprendizaje del lenguaje comienza con el análisis acústicofonológico del habla, por lo que, parece lógico pensar que el córtex auditivo primario, y sus áreas adyacentes, es el candidato neurológico para realizar 
este proceso. Consecuentemente, muchas investigaciones sobre el origen neuroanatómico de la Dislexia han dirigido su atención a esta área encontrando importantes diferencias neuronales y anatómicas con los lectores normales. La relación entre el tronco encefálico auditivo, el procesamiento temporal de los sonidos del habla y de este con una anormal discriminación cortical y habilidades lectoras, es relativamente reciente (Banai, Nicol, Zecker y Kraus, 2005).

El núcleo medial geniculado (NMG) es el equivalente auditivo al NGL visual, situado también en el tálamo junto a este último. Para Díaz, Hintz, Kiebel, y von Kriegstein (2012), el déficit fonológico en los disléxicos tiene su origen en un mal funcionamiento del mecanismo neuronal que ajusta dinámicamente el NGM de acuerdo con las predicciones de las áreas corticales (de arriba abajo) para optimizar el procesamiento del habla.

Ahveninen, Jääskeläinen, Raij, Bonmassar, Devore et al (2006) identificaron también una doble vía que procesa la información auditiva de forma paralela: la primera vía procesa la identidad del habla y el sonido y representa el "qué" (en el giro de Heschl, giro temporal y planum polare); la segunda vía identifica la ubicación de procedencia del sonido y representa el "dónde" (en planum temporale y giro temporal superior). La vía "dónde " se activa 30 ms. antes que la vía "qué ", lo que posibilita al cerebro usar información espacial de arriba a abajo en la percepción del objetivo auditivo. Es importante destacar que el lóbulo parietal y frontal derechos mostraron mayor activación durante la tarea de localización que durante la tarea de identificación del fonema. Por lo tanto, también en el procesamiento auditivo parece que la vía "dónde" guía a la vía "qué" para la identificación del objetivo auditivo.

El giro de Heschl, es el principal receptor de la información auditiva proveniente del NGM. Se activa durante el procesamiento auditivo para tareas de tono y semánticas (Warrier, Wong, Penhune, Zatorre, Parrish et al, 2009). Es en esta zona donde se codifican las características temporales del habla, como el tiempo de inicio de la voz (Hornickel, Skoe, Nicol, Zecker y Kraus, 2009). Clark, Helland, Specht, Narr, Manis, Toga, y Hugdahl (2014) encontraron diferencias estructurales en esta zona del hemisferio izquierdo en niños que más tarde desarrollaron Dislexia, antes del aprendizaje de la lectura.

Con la combinación de las técnicas de neuroimagen y las de emisión de oscilaciones electromagnéticas de los distintos tipos de frecuencia (alfa entre 8-13 Hz, beta entre $12 \mathrm{~Hz}-30 \mathrm{~Hz}$, gamma entre 25 - $100 \mathrm{~Hz}$, delta entre 1-3 Hz y theta $3.5-7.5 \mathrm{~Hz}$ ) es posible observar las zonas del cerebro que se activan con distintos tipos de estímulos sonoros y rangos de frecuencia. Las oscilaciones corticales a diferentes tasas temporales produ- 
cen información acústica relevante para la estructura fonológica del habla, con información de banda delta relacionada con la extracción de patrones de estrés silábico, información de banda theta relacionada con la extracción de información silábica, información de la banda beta relacionada con las unidades de inicio y rima (para dividir una sílaba en unidades lingüísticas de tiempo de inicio, segmento marcado por la vocal) e información de banda baja gamma relacionada con la información fonética (Cutini, Sz cs, Mead, Huss y Goswami, 2016). Los hemisferios están especializados en tipos de frecuencia distintos e interaccionan para integrar la información de las distintas frecuencias. El derecho está especializado en oscilaciones delta y theta y el izquierdo en oscilaciones gamma. Como sugieren Cutini et al (2016), las diferencias en la especialización hemisférica encontradas en los disléxicos (Lizarazu, Lallier, Molinaro, Bourguignon, Paz-Alonso et al, 2015; Lehongre, Morillon, Giraud y Ramus, 2013) para las ondas gamma podrían surgir durante el aprendizaje de la asociación grafema-fonema, cuando se está integrando en las representaciones fonológicas neurales, es decir, puede deberse a un efecto compensatorio, por lo que podría ser una consecuencia y no la causa de la pobre conciencia fonológica en disléxicos.

Por el contrario, es el procesamiento temporal del habla lo que parece estar detrás de la conciencia fonológica. El habla contiene estructuras temporales o rítmicas que el cerebro analiza para permitir el procesamiento lingüístico (Overath, McDermott, Zarate y Poeppel, 2015). Banai, Hornickel, Skoe, Nicol, Zecker y Kraus (2009) encontraron que el ritmo deficiente de codificación auditiva en esta área y la pobre representación de la señal armónica son características de los niños que leen mal y puntúan bajo en tareas de conciencia fonológica y deletreo, mientras que los buenos lectores se caracterizan por una codificación más precisa en términos de tiempo y una representación más robusta de los armónicos del habla.

Otro concepto importante para la línea de investigación sobre la percepción del habla en disléxicos, también relacionado con el procesamiento temporal, es el Speech-brain entrainment o phase entrainment (alineación de la actividad neuronal a estímulos de fluctuaciones temporales) que ha resultado ser crítico para la compresión del habla acústica (Riecke, Formisano, Sorger, Ba kent y Gaudrain, 2018). Los niños con un funcionamiento atípico de esta phase entrainment podrían, según Cutini et al (2016), procesar la señal del habla de una forma diferente a los niños con desarrollo neurotípico, y esto antes de comenzar con el aprendizaje de la lectura por este déficit de modulación en el NMG, apuntado más arriba.

La discriminación auditiva se reconoce como uno de los elementos cruciales en el desarrollo del habla y la lectoescritura. Un aspecto importante de la discriminación auditiva es la capacidad de percibir el habla separándola 
de otros estímulos ruidosos presentes en el ambiente. Chandrasekaran, Hornickel, Skoe, Nicol y Kraus (2009) descubrieron que los niños con Dislexia presentan déficits en el tronco encefálico para la descodificación dependiente del contexto de las características del habla. Vidyasagar y Pammer (2010) apuntan que, este déficit puede ser explicado también por la teoría de vía dorsal, que guía la atención espacial para localizar la procedencia del objetivo auditivo. Una deficiente conexión entre la vía occipito-parietal que guía la atención espacialmente y el tálamo, responsable de descartar los estímulos no relevantes y codificar la información espacial (Pulvinar), así como, el sistema magnocelular que permite el procesamiento rápido, explicaría por tanto, también los déficits auditivos que podrían ser incluidos en un déficit del procesamiento sensorial espacio-temporal más amplio.

En conclusión, las dimensiones de espacio y tiempo están presentes trasversalmente y de forma inconsciente (solo la vía ventral produce percepciones accesibles a la conciencia) en el aprendizaje de la lectura y la escritura. En la Tabla 1 señalamos estas dos dimensiones con respecto a algunos aspectos de la lectura.

\section{TABLA 1}

Aspectos espaciotemporales de la lectura

\begin{tabular}{|c|c|}
\hline Dimensión espacial & Dimensión temporal \\
\hline $\begin{array}{l}\text { - Posición relativa de las letras en la pa- } \\
\text { labra }\end{array}$ & $\begin{array}{l}\text { - Secuenciación de los estímulos auditivos } \\
\text { y visuales }\end{array}$ \\
\hline - Dirección de las letras & $\begin{array}{l}\text { Características temporales del habla: } \\
\text { comienzo y fin de sílabas y palabras (con- } \\
\text { ciencia fonológica) }\end{array}$ \\
\hline - Dirección de lectura de izquierda-derecha & $\begin{array}{l}\text { - Sincronización visual y auditiva de los } \\
\text { estímulos (asociación de estímulos) }\end{array}$ \\
\hline \multicolumn{2}{|l|}{ - Saltos de línea } \\
\hline \multicolumn{2}{|l|}{$\begin{array}{l}\text { - Atención a localización espacial de la } \\
\text { procedencia del sonido (discriminación } \\
\text { auditiva) }\end{array}$} \\
\hline
\end{tabular}

La correcta sincronización temporal de los estímulos visuales y auditivos hace posible su asociación e integración. En este proceso la atención (guiada desde arriba) a estas dimensiones espaciotemporales por parte de las áreas primarias auditivas y visuales modula las neuronas de las áreas sensoriales primarias (abajo) que envían la información procesada a áreas corticales superiores para su correcto almacenamiento, lo que facilita el reconocimiento ante el mismo estímulo en el futuro. La atención espacial afecta no solo a la habilidad para detectar el estímulo, sino también para representar mentalmente este estimulo (Quercia, Feiss y Michel, 2013) y, por tanto, para almacenarlo y recuperarlo. 


\section{PROPUESTA DIFERENCIADORA Y CONSECUENCIAS COGNITIVAS DEL DÉFICIT DEL PROCESAMIENTO SENSORIAL ESPACIO-TEMPORAL}

La Federación Española de Dislexia (FEDIS) incluye en el perfil de los disléxicos síntomas relacionados con la desorientación espacio-temporal, la torpeza visomotora, el déficit en la memoria serial, el esquema corporal y la lateralización (para más detalle en relación a estos síntomas véase Etchepareborda, 2002). Los déficits neuroanatómicos auditivos y visuales detectados en gran parte de los sujetos disléxicos, explican estas manifestaciones por un efecto cascada desde el tálamo hacia el lóbulo parietal que a su vez regula la actividad de la vía ventral. Las consecuencias cognitivas de un déficit el procesamiento de la información espacio-temporal se extiende más allá de los procesos involucrados en la lectoescritura.

El tiempo es percibido como una estructura secuencial lineal de eventos de duración determinada formados por memorias visuales, auditivas, semánticas y otras que procesamos en paralelo y que, integradas, les dan identidad propia, forman esquemas de eventos que nos permiten anticipar otros (Zacks, Speer, Swallow, Braver, y Reynolds, 2007). Por ejemplo, el día de ayer lo recordamos como una serie de actividades ordenadas secuencialmente formado por imágenes, palabras y acciones (mañana, sueño, desayuno, tarde, media hora, sol, pronto, levantarse, cena), sonidos, emociones e interacciones con otras personas y objetos en determinados momentos a lo largo del día. Para integrar todas estas percepciones que sitúan un evento en un momento y duración determinados, el procesamiento en paralelo de las informaciones sensoriales y su correspondiente integración en la memoria debe ser eficiente e integrado, lo que precisa de atención a los detalles espaciales, percepción visual consciente y memoria visual y semántica (Grossberg, 1999). Un concepto relacionado con la percepción temporal es la "memoria prospectiva" cuyo déficit se ha encontrado en adultos disléxicos cuando la declaración de intenciones se basa en pistas temporales y/o cuando tienen que ser auto-iniciadas sin que los eventos ambientales puedan promoverlas (Smith-Spark, Zi cik, y Sterling, 2017).

El déficit fonológico en el caso de los niños con un déficit en el procesamiento audiovisual, parece ser una consecuencia de éste último. Como apuntábamos más arriba, el córtex parietal posterior conectado al área sensorial primaria a través de la vía dorsal, recibe información del córtex auditivo que integra con la información visual, lo que permite asociar ambos tipos de estímulos (grafema-fonema, color-nombre, nota musical-sonido). Esta integración se dificulta si cualquiera de los dos tipos de imputs, visuales 0 auditivos, no se recibe correctamente (Vidyasagar y Pammer, 2010). Por ejemplo, Kronschnabel, Brem, Maurer y Brandeis (2014) defienden que el 
déficit audiovisual en los adolescentes disléxicos de la muestra de su estudio puede explicar el déficit en la conciencia fonológica mediante la deficiente integración a nivel fonético. Este déficit audiovisual para el procesamiento de la información espacio-temporal es la causa de un lento reconocimiento de las letras y una pobre habilidad para secuenciar las letras y sus sonidos que, a su vez, deriva de un déficit en sincronización sensorial (sensory timing) de los imputs visuales durante la lectura y los sonidos de la palabra cuando se escuchan, esto es, un déficit en el procesamiento temporal (Stein, 2018). El déficit en el procesamiento temporal del habla oral también es explicado por la por la teoría magnocelular, puesto que este contribuye a la rápida integración de la información visual mientras leemos permitiendo un procesamiento eficiente, sincrónico, de la información visual espacial y temporal y puede intervenir con un papel equivalente con la información auditiva (Quercia, Feiss y Michel, 2013).

La existencia de estos problemas relacionados con el déficit visoespacial en los disléxicos se ha encontrado en un porcentaje entre el 30\% y el 65\% (Elliot y Grigorenko, 2014). Otro estudio que da cuenta de la heterogeneidad de lo que Ilamamos "disléxicos" es el de Menguini, Carlesimo, Marotta, Finzi y Vicari (2010), en el que se propusieron como objetivo de su estudio evaluar si el déficit de aprendizaje en los disléxicos se limita solo a componentes verbales de la capacidad de memoria a largo plazo o también están implicados los de los objetos visuales y el área viso-espacial. El porcentaje de sujetos que mostró solo un déficit fonológico fue del 18,3\%, mientras que el 76,6 \% presentaba además otros déficits cognitivos asociados.

Por tanto, si bien se ha demostrado que el déficit del procesamiento visoespacial y auditivo es una característica de muchos niños con problemas en la lectoescritura no es generalizable a todos los niños con esta dificultad, por lo que sería lógico platear que estamos ante trastornos diferentes que pueden o no darse juntos (Elliot y Grigorenko, 2014).

Todo lo expuesto nos lleva a plantear dos grandes perfiles distintos: 1) sujetos con déficit lectoescritor de origen multifactorial; 2) sujetos cuyo trastorno incluye el déficit lectoescritor como consecuencia de problemas relacionados con el procesamiento de la información espacio-temporal sin relación con el QI. Por otra parte, este grupo podría ser el que acusa una Dislexia mixta, que es la mayoritaria (Vidyasagar y Pammer, 2010). La figura siguiente es una representación gráfica de esta propuesta reinterpretativa.

El circuito occipital-parietal, uno de los circuitos de la vía dorsal, transforma las representaciones visuales en referencias a las partes del cuerpo y el ojo, lo que explica la descoordinación visomotora observada en los niños con este trastorno. Esta región proporciona información sobre 


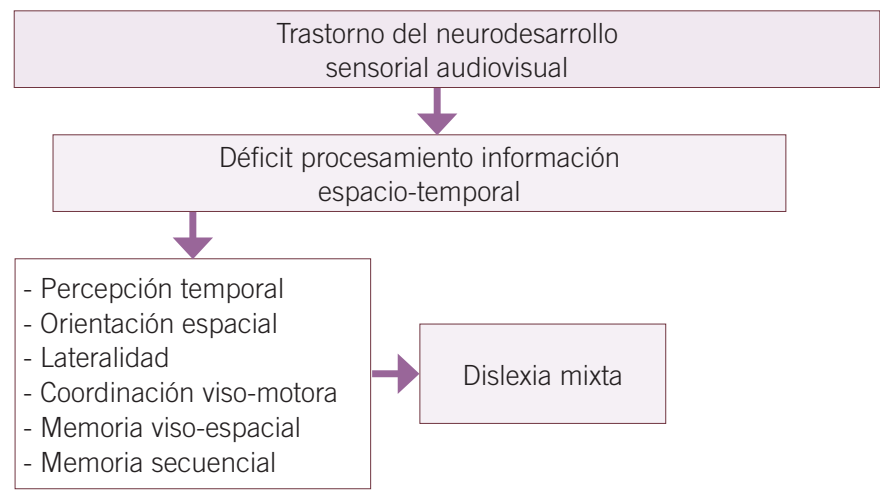

aspectos egocéntricos de la visión, incluida la iniciación y el control de los movimientos sacádicos, que son cruciales para la orientación y la memoria de trabajo espacial. De acuerdo con de Freitas, Pedâo y Barela (2014), sorpresivamente, la habilidad del procesamiento visomotor y su incidencia en la Dislexia ha sido poco investigada hasta hace poco. En los últimos años han comenzado a aparecer estudios relativos al control postural, el equilibrio o la propiocepción y su incidencia en disléxicos (Quercia, Seigneuric, Chariot, Vernet, Pozzo et al (2005).

La intervención en este tipo de sujetos sobre la conciencia fonológica también puede mejorar el rendimiento en la lectura, pero no porque se esté actuando sobre la causa del déficit, sino como consecuencia de la mayor exposición auditiva y visual a las letras, sílabas y palabras, lo que permite el entrenamiento en la búsqueda, reconocimiento y memoria visual de la situación espacial relativa de cada la letra dentro de la palabra y la integración de los estímulos visuales y auditivos.

En conclusión, los efectos cognitivos del déficit en el procesamiento espaciotemporal de los estímulos visuales y auditivos no se limitan en este grupo al que se refiere FEDIS a la percepción del habla, sino a la interiorización del tiempo, en general. Tampoco la orientación espacial afecta en este grupo solo a la inversión de las letras, a la situación de las letras en las palabras y de las palabras en las oraciones, sino a cualquier actividad o proceso en el que esté implicada la orientación en el espacio peripersonal, que tiene como consecuencia secundaria el déficit en la lateralidad, la direccionalidad y el eje corporal que conduce a una descoordinación visomotora. 


\section{IMPLICACIONES PARA UNA DIDÁCTICA INCLUSIVA}

Las consecuencias cognitivas de este trastorno del neurodesarrollo visual y/o auditivo no suelen ser tenidas en consideración por los maestros que entienden la Dislexia como una dificultad en la lectoescritura cuya principal causa es el déficit de la conciencia fonológica. Sin embargo, afectan a la eficiente realización de una gran cantidad de tareas cotidianas del aula sin que se reconozca por parte de los maestros como una consecuencia de este trastorno y que, por tanto, el alumno que lo padece no es capaz de evitar hasta que su cerebro consigue compensar esas deficiencias. En Educación Infantil estas consecuencias cognitivas se manifiestan en la falta de habilidad en actividades cotidianas de forma inesperada, es decir, no se corresponde con la normalidad en el desarrollo de otras áreas. Durante la etapa prelectora, los niños que se encuadran en este trastorno del neurodesarrollo pueden ser detectados a través de manifestaciones relacionadas con ciertas actividades escolares y extraescolares como las que se relacionan en la Tabla 1.

\section{TABLA 1}

\section{Consecuencias cognitivas y tareas asociadas al déficit espacio-temporal}

\section{Consecuencia cognitiva}

- Interiorización de conceptos relacionados con el tiempo

- Orientación y memoria espacial

- Equilibro, coordinación psicomotriz, propiocepción, lateralidad

- Atención y búsqueda visual

- Memoria secuencial

- Asociación del estímulo visual con su nombre 0 sonido
Algunas tareas asociadas

- Aprender conceptos como: mañana-tarde, meriendacena, anteayer, pasado mañana, etc.

- Aprender las horas del reloj

- Ejecutar una tarea en el tiempo adecuado

- Organización (olvidan el lugar donde se guardan las cosas o dónde las han puesto, no calculan correctamente el espacio disponible)

- Identificar notas musicales por su situación en el pentagrama

- Atarse los cordones de las zapatillas, abotonarse el babi, bajar las escaleras corriendo, montar en bicicleta, golpear o agarrar la pelota, columpiarse solo, seguir el ritmo de las canciones

- Realizar los trazos que forman las letras y los números (escritura en espejo de números y letras)

- Aprender conceptos relacionados con el espacio: arribaabajo, delante-detrás, los puntos cardinales, etc.

- Imitar dibujos a partir de un modelo

- Localizar en la pizarra o en las paredes del aula la información

- Relatar hechos siguiendo una secuencia temporal lógica

- Memorizar abecedario, estaciones, días de la semana, meses del año, tablas de multiplicar, números de teléfono

- Seguir órdenes encadenadas, memorizar poemas o canciones

- Reconocer y asociar con su nombre colores, formas, letras, notas musicales y números

- Reconocer las caras de sus compañeros en las fotos del mural 
Esta relación no pretende ser exhaustiva, sino evidenciar que existe una relación directa entre el origen neurológico del trastorno, las consecuencias cognitivas que de él se derivan y algunas de las tareas que para estos niños entrañan una gran dificultad mientras que otros niños aprenden a realizarlas de forma natural.

En Educación Primaria algunas actividades entrañan habilidades cognitivas que, erróneamente, en el caso de estos niños, se suponen adquiridas y que están relacionados con la percepción espacio-temporal y la memoria secuencial. Los maestros pueden desconocer que su incapacidad en el desempeño de estas actividades se relaciona con un trastorno si su atención se centra únicamente en la lectoescritura:

- Búsqueda de palabras en el diccionario: implica, además de la asociación grafema-fonema que puede ya estar adquirida, la retención en la memoria de trabajo espacial el lugar que ocupa cada letra en la palabra a localizar y la percepción del lugar que ocupan las letras en las palabras del diccionario.

- Realizar ejercicios en el cuaderno: ordenar los ejercicios y sus respuestas en el cuaderno requiere un sentido topográfico, es decir, orientación espacial, dimensionar el espacio disponible correctamente, elegir un lugar adecuado para cada uno de los elementos y calibrar correctamente el tamaño de cada uno de ellos anticipando el resultado mentalmente. Como consecuencia, el cuaderno de los disléxicos está desordenado, el tamaño de las letras y números es irregular, los espacios están desaprovechados 0 , por el contrario, sobrecargados o insisten en escribir solo por la cara que con el cuaderno abierto queda a su derecha por la desatención al campo visual izquierdo (Quercia, Feiss y Michel, 2013).

- Redacción: implica ordenar las ideas siguiendo una secuencia temporal lógica además de los procesos relacionados con la escritura.

- La evaluación del idioma inglés: la evaluación en esta asignatura suele dividirse en tres tareas distintas: listening, speaking y writing. La forma más habitual de evaluar el listening consiste en la audición de una sentencia reproducida por un reproductor de CD después de la cual el alumno debe marcar en una ficha la imagen o el texto correcto con la que se corresponde la sentencia. Esta tarea exige, además de una eficiente discriminación auditiva y conciencia fonológica, una búsqueda visual y el reconocimiento de la imagen o el texto en la ficha (normalmente en blanco y 
negro y defectuosa debido al fotocopiado) que se corresponde con la audición. En cuanto al speaking, la forma habitual de evaluarlo es realizar preguntas al alumno de forma oral que éste responde. Exige pues la discriminación auditiva y la traducción mental simultánea seguida de la elección mental de la respuesta correcta, la traducción mental al inglés y la dicción de la misma. El alumno disléxico necesitará que la pregunta sea repetida varias veces puesto que no retendrá en la memoria de trabajo la secuencia de las palabras pronunciadas y será más lento en la recuperación de la memoria del significado de las palabras. Una vez haya traducido la pregunta deberá seleccionar la respuesta, para lo que requiere recuperar la secuencia correcta para ordenar las palabras (sintaxis) de su respuesta. Respecto al writing, los disléxicos tienden a escribir la palabra tal como suena debido a su déficit de asociación entre sonidos y su representación gráfica, pero también por su déficit en la memoria secuencial visual que permite recordar la palabra como un todo.

- En Matemáticas, y dado que no existe relación entre este trastorno y QI, estos alumnos con inteligencia normal o alta no tendrán dificultad para entender los conceptos matemáticos. Es frecuente que piense un número y escriba otro, o que coloque los números desordenados en las operaciones dificultando su resolución, o que se le olvide multiplicar uno de los multiplicadores por su dificultad para seguir una secuencia. Debemos, por tanto, valorar el proceso y el resultado de la operación (objetivo conceptual y objetivo procedimental) de forma diferenciada. Las tablas de multiplicar constituyen secuencias de números; las memorizamos recordando el orden relativo de cada cifra con respecto al anterior y al posterior. Es inútil y frustrante para estos alumnos intentar que las memoricen al mismo ritmo que el resto, y si lo hacen, probablemente las olvidarán antes que los demás. Deberán ser facilitadas cuando constituyan una barrera para el resto de aprendizajes y en las pruebas de evaluación, pudiendo ponderar el objetivo "memorizar las tablas de multiplicar" de forma separada a las resolución de problemas.

En cuanto a las operaciones aritméticas como sumas, restas y divisiones, una de las mayores dificultades con las que se encuentran los disléxicos es recordar el orden en que se realizan los distintos pasos de la operación, puesto que se trata de una secuencia en la que además la direccionalidad es esencial. A menudo no recuerdan si la operación comienza por la derecha/ izquierda, por arriba/abajo. 
Si los métodos de enseñanza en cursos anteriores han sido los adecuados, el alumno con este trastorno no tendrá dificultad en resolver problemas mentalmente (objetivo conceptual). Es decir, entenderá la situación problemática, los conceptos incluidos en el enunciado del problema y las operaciones que precisa para resolver el problema (objetivo procedimental). La ayuda del profesor en estos casos deberá centrarse en la planificación de la secuencia de estrategias para la resolución y en recordar la secuencia y direccionalidad de las operaciones necesarias.

- Música: la dificultad de estos alumnos en la asociación de una representación simbólica y su correspondencia con un sonido se extiende también al lenguaje musical. En el caso de estos niños, esa asociación se complica al depender la identificación de la situación de la nota en el pentagrama (búsqueda visual y situación espacial). Además, es necesaria la coordinación visomotora para la ejecución. Por otra parte, una melodía constituye una secuencia de notas, por lo que será más complicado para estos alumnos recordarla.

- Geografía: una de las tareas más dificultosas para estos alumnos es situar en un mapa montañas y ríos, puesto requiere la orientación y la memoria viso-espacial.

- En Educación Física dos de los criterios a evaluar en los primeros años de primaria es el equilibrio (que precisa información vestibular sobre la posición de los brazos, el ojo y la cabeza) y los juegos de pelota (movimientos de alcance y agarre).

- Para que la agenda escolar constituya una herramienta útil para la organización de la preparación de exámenes o entrega de trabajos, el alumno necesita haber interiorizado los días de la semana y los meses que le permite situarse en el tiempo, en qué día y mes se encuentra, anotar la fecha de entrega en el día correspondiente y de cuánto tiempo dispone para realizar y entregar las tareas. Este tipo de alumnos necesitan más tiempo para anotar en su agenda las indicaciones de este tipo del que a menudo no dispone, por lo que más que una herramienta útil, es una tarea que le produce confusión.

En definitiva, los conocimientos neurocognitivos nos ayuda a los educadores a comprender las dificultades en la adquisición de ciertas habilidades que están implicadas en la ejecución de tareas cotidianas del aula, posibilitando el diseño de una didáctica que tenga en consideración esas dificultades. 


\section{BIBLIOGRAFÍA}

Ahveninen, J.; liro P. Jääskeläinen, I.P. ; Raij, T; Bonmassar, G.; Devore, S. ; Hämäläinen, M; Levänen, S.; Lin, F-H.; Sams, M.; Shinn-Cunningham, B. G.; Witzel, T. y Belliveau, J. W. (2006) Task-modulated "what" and "where" pathways in human auditory cortex. PNAS, 103(39), 14608-14613 https://doi.org/10.1073/pnas.0510480103

Banai, K.; Hornickel, J.; Skoe, E.; Nicol, T.; Zecker, S. y Kraus, N. (2009). Reading and subcortical auditory function. Cerebral Cortex, 19, 2699-2707 https://doi.org/10.1093/cercor/ bhp024

Banai, K.; Nicol, T.; Zecker, S.G. y Kraus, N. (2005). Brainstem timing: implications for cortical processing and literacy. Journal of Neuroscience, 25(43), 9850-9857. doi: 10.1523/ JNEUROSCI.2373-05.2005

Ben-Shachar, M., Dougherty, R. F., Deutsch, G. K., y Wandell, B. A. (2007). Contrast Responsivity in MT+ Correlates with Phonological Awareness and Reading Measures in Children. Neurolmage, 37(4), 1396-1406. http://doi.org/10.1016/j.neuroimage.2007.05.060

Bueti, D.; Bahrami, B. y Walsh, V. (2008). Sensory and associative cortex in time perception. Journal of Cognitive Neuroscience, 20, 1054-1062. doi:10.1162/jocn.2008.20060

Clark, K.A.; Helland, T; Specht, K.; Narr, K.L.; Manis, F. R.; Toga, A.W. y Hugdahl, K. (2014) Neuroanatomical precursors of dyslexia identified from pre-reading through to age 11, Brain, 137(12), 3136-3141, https://doi.org/10.1093/brain/awu229

Cutini, S.; Sz cs, D; Natasha Mead, N; Huss, M y Goswami, U. (2016). Atypical right hemisphere response to slow temporal modulations in children with developmental dyslexia. Neurolmage, 143, 40-49 http://dx.doi.org/10.1016/j.neuroimage.2016.08.012

de Freitas, P.B.; Pedão, S.T. y Barela, J.A. (2014) Visuomotor processing and hand force coordination in dyslexic children during a visually guided manipulation task. Research in Developmental Disabilities. 35(10):2352-2358. doi: 10.1016/j. ridd.2014.06.002..

Díaz, B., Hintz, F., Kiebel,S.J. y von Kriegstein, K.(2012). Dysfunction of the auditory thalamus in developmental dyslexia. PNAS, 109(34), 13841-13846. doi: 10.1073/ pnas. 1119828109.

Elliott, J.G. y Grigorenko, E.L. (2014). The Dyslexia Debate. Nueva York: Cambridge University Press.

Etchepareborda, M.C. (2002) Detección precoz de la Dislexia y enfoque terapéutico. Revista de Neurología, 34 (Supl1), 13-23. Recuperado: https://pdfs.semanticscholar.org/2812/ bb248b85f9d7afe38996c79cb5e80ea610cc.pdf

Fischer, J. y Whitney, D. (2009) Precise Discrimination of Object Position in the Human Pulvinar. Human Brain Mapping, 30, 101-111, Doi: 10.1002/hbm.20485

Franceschini S, Gori S, Ruffino M, Pedrolli K, Facoetti A. (2012) A causal link between visual spatial attention and reading acquisition. Current Biology, 22(9), 814-819. doi: 10.1016/j.cub.2012.03.013.

Friederici, A. D. (2011) The brain basis of language processing: from structure to function. Physiological Reviews, 91, 1357-1392, doi:10.1152/physrev.00006.2011 
Ghosh, S.; Basu, A.; Kumaran, S. S. y Khushu, S. (2010). Functional mapping of language networks in the normal brain using a word-association task. The Indian Journal of Radiology \& Imaging, 20(3), 182-187. http://doi.org/10.4103/0971-3026.69352

Giraldo-Chica, M.; Hegarty, J.P. y Schneider, KA. (2015). Morphological differences in the lateral geniculate nucleus associated with dyslexia. Neuroimage Clin. (20)7, 830-836. doi: 10.1016/j.nicl.2015.03.011

Gori, S.; Seitz, A.R.; Ronconi, L.; Franceschini, S. y Facoetti, A. (2016). Multiple Causal Links Between Magnocellular-Dorsal Pathway Deficit and Developmental Dyslexia. Cerebral Cortex, 26, 4356-4369. https://doi.org/10.1093/cercor/bhv206

Grossberg, S. (1999) The link between brain learning, attention, and consciousness. Consciousness and Cognition,8(1), 1-44. https://doi.org/10.1006/ccog.1998.0372

Hornickel, J.; Skoe, E.; Nicol, T.; Zecker, S. y Kraus, N. (2009). Subcortical differentiation of stop consonants relates to reading and speech-in-noise perception. PNAS, 106, 13022-13027. doi: 10.1073/pnas.0901123106

Kozlovskiy, S.A.; Pyasik, M.M.; Korotkova, A.V.; Vartanov, A.V.; Glozman, J.M. y Kiselnikov, A.A. (2014). Selective involvement of lingual gyrus in working memory and perception of different types of visual stimuli. Journal of the International Neuropsychological Society, 20, (S2), 43-43

Kravitz, D. J.; Kadharbatcha, S. S.; Baker, C.I. y Mishkin, M. (2011) A new neural framework for visuospatial processing. Nat Rev Neurosci 12(4):217-230. doi: 10.1038/nrn3008

Kronschnabel J.; Brem S.; Maurer U. y Brandeis D. (2014) The level of audiovisual print-speech integration deficits in dyslexia Neuropsychologia, 62, 245-61. doi: 10.1016/j. neuropsychologia.2014.07.024

Lehongre, K.; Morillon B.; Giraud, A-L. y Ramus, F (2013). Impaired auditory sampling in dyslexia: further evidence from combined fMRI and EEG Frontiers in Human Neurosciencie. https://doi.org/10.3389/fnhum.2013.00454

Livingstone, M.S.; Rosen, G.D.; Drislane, F.W. y Galaburda, A.M. (1991). Physiological and anatomical evidence for a magnocellular defect in developmental dyslexia. PNAS, 88, 7943-7947.

Lizarazu, M.; Lallier, M.; Molinaro, N.; Bourguignon, M.; Paz-Alonso, P.M.; Lerma-Usabiaga, G y Carreiras, M. (2015). Developmental evaluation of atypical auditory sampling in dyslexia: Functional and structural evidence. Human Brain Mapp. 36(12), 49865002. doi: 10.1002/hbm.22986.

Lobier, M.A.; Peyrin, C.; Pichat, C.; Le Bas, J.F. y Valdois, S. (2014) Visual processing of multiple elements in the dyslexic brain: evidence for a superior parietal dysfunction. Frontiers in Human Neurosciencie, 8, 479. doi:10.3389/fnhum.2014.00479

Menghini, D.; Carlesimo, G. A.; Marotta, L.; Finzi, A.; Stefano Vicari, S. Developmental dyslexia and explicit long term memory (2010). Dyslexia, 16(3), https://doi.org/10.1002/dys.410

Mishkin, M.; Ungerleider, L. G. y Macko, K. (1983). Object vision and spatial vision_two cortical pathways. Trends In Neurosciences, 6, 414- 417.

Mitchell, A.S. ;Sherman, S. M.; Sommer, M.A.; Mair, R. G.; Vertes, R. P. y Chudasama, Y. (2014). Advances in Understanding Mechanisms of Thalamic Relays in Cognition and Behavior. Journal of Neuroscience, 34(46), 15340-15346. doi: https://doi. org/10.1523/JNEUROSCI.3289-14.2014 
Müller-Axt, C.; Anwander,A. y von Kriegstein, K. (2017) Altered structural connectivity of the left visual thalamus in developmental dyslexia Current Biology. 27(23), 3692-3698. doi: 10.1016/j.cub.2017.10.034

Overath, T; McDermott, T. J; Zarate, J.M. y Poeppel, D. (2015) The cortical analysis of speech-specific temporal structure revealed by responses to sound quilts. Nature Neuroscience, 18, 903-911 doi:10.1038/nn.4021

Pennington, B.F. (2006) From single to multiple deficit models of developmental disorders. Cognition, 101, 2, 385-413 https://doi.org/10.1016/j.cognition.2006.04.008

Quercia, P., Feiss, L. y Michel, C. (2013). Developmental dyslexia and vision. Clinical Ophthalmology, 7, 869-881. http://doi.org/10.2147/OPTH.S41607

Quercia, P.; Seigneuric, A.; Chariot, S. ; P. Vernet, P ; Pozzo, T. ; Bron, A. ; C. Creuzot-Garcher, C. y Fobichon, F. (2005). Proprioception oculaire et dyslexie de développement. À propos de 60 observations cliniques. Journal Français D'Ophtalmologie, 28(7), 713-723

Riecke, L; Formisano, E; Sorger, B; Ba kent, D y Gaudrain, E (2018) Neural Entrainment to Speech Modulates Speech Intelligibility Current Biology. 28(2):161-169. doi: 10.1016/j.cub.2017.11.033.

Rolls, E. T. (2004), Convergence of sensory systems in the orbitofrontal cortex in primates and brain design for emotion. Anatomical Record, 281A, 1212-1225. doi:10.1002/ ar.a.20126

Smith-Spark, J. H.; Zi cik, A. P. y Sterling, C. (2017). Adults with developmental dyslexia show selective impairments in time-based and self-initiated prospective memory: Selfreport and clinical evidence. Research in Developmental Disabilities, 62, 247-258. https://doi.org/10.1016/j.ridd.2016.12.011

Stein, J. (2018). What is Developmental Dyslexia? Brain Sciences, 8(2), 26; doi:10.3390/ brainsci8020026

Stoeckel, C.; Gough PM; Watkins K.E. y Devlin J.T. (2009). Supramarginal gyrus involvement in visual word recognition Cortex, 45(9), 1091-1096. doi: 10.1016/j.cortex.2008.12.004.

Twomey, T.; Kawabata Duncan, K ; Price, C.J. y Devlina, J.T. (2011) Top-down modulation of ventral occipito-temporal responses during visual word recognition Neurolmage, (55)3, 1242-1251 https://doi.org/10.1016/j.neuroimage.2011.01.001

Vidyasagar, T.R y Pammer, K. (2010) Dyslexia: a deficit in visuo-spatial attention, not in phonological processing. Trends in Cognitive Sciences, 14(2), 57-63 doi:10.1016/j. tics.2009.12.003

Warrier, C; Wong, P.; Penhune, V.; Zatorre, R.; Parrish, T.; Abrams, D y Kraus, N. (2009). Relating Structure to Function: Heschl's Gyrus and Acoustic Processing. The Journal of Neuroscience, 29(1), 61-69. doi: 10.1523/JNEUROSCI.3489-08.2009

Weyand, T. (2015). The multifunctional lateral geniculate nucleus. Reviews in the Neurosciences, 27(2), 135-157. doi:10.1515/revneuro-2015-0018

Zacks, J. M., Speer, N. K., Swallow, K. M., Braver, T. S. y Reynolds, J. R. (2007). Event Perception: A Mind/Brain Perspective. Psychological Bulletin, 133(2), 273-293. http://doi.org/10.1037/0033-2909.133.2.273 